\title{
Call for Special Issue Papers: Drug Delivery Research
}

\author{
Deadline for Manuscript Submission: August 1, 2020
}

Guest Editor: Harsh Chauha, PhD; Special Editor of Drug Delivery Research, ASSAY and Drug Development Technologies

Advent of in silico screening techniques and high throughput technologies have resulted in synthesis of highly potent compounds with challenging physicochemical properties. Also, there are several issues related to the formulation of current marketed and repurposed drugs. For these challenging compounds, delivery systems are designed to enhance solubility/ stability, target specific sites in the human body, optimize drug therapy, improve PK-PD profile and reduce side/ adverse effects. The special issue focuses on recent advancement in the area of formulation and drug delivery technologies (solutions, semi-solids, nanoparticles, liposomes, emulsions, amorphous dispersions etc.) for challenging drugs.

Special Issue Editor Dr. Harsh Chauhan will consider articles from the full breadth of research in the field from original research papers, reviews, and methods papers, to perspectives, profiles, commentaries, and exemplary front matter.

Areas of interest include, but are not limited to:

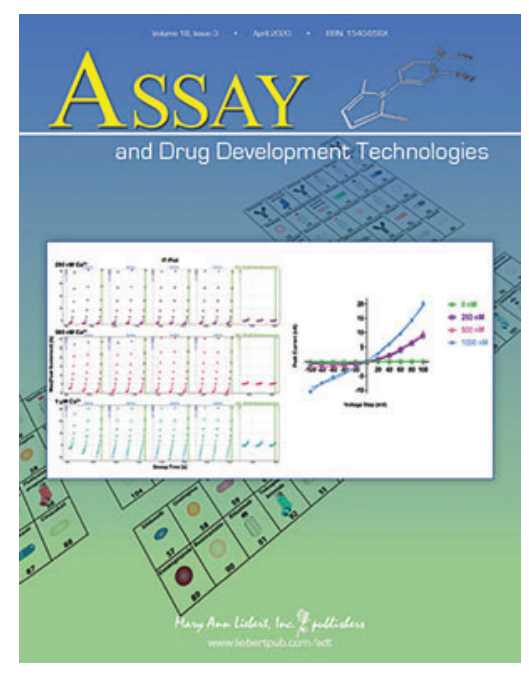

- Advancement in novel drug delivery technologies.

- Formulation, characterization, in vitro/in vivo studies of nanoparticles.

- Solubilization and delivery of poorly soluble compounds.

- Targeted drug delivery systems.

- Advancement in topical/ transdermal drug delivery systems.

Contributions will receive prompt and thorough peer review. Please refer to our Instructions for Authors at www.liebertpub.com/adt before submitting your manuscript for consideration. Authors are encouraged to vet ideas directly with Dr. Chauhan via e-mail (harshchauhan@creighton.edu) before submitting.

ASSAY and Drug Development Technologies provides access to novel techniques and robust tools that enable critical advances in earlystage screening. This research published in the Journal leads to important therapeutics and platforms for drug discovery and development. This reputable peer-reviewed journal features original papers application-oriented technology reviews, topical issues on novel and burgeoning areas of research, and reports in methodology and technology application.

Manuscript Submission Deadline: August 1, 2020

Visit the Instructions for Authors: www.liebertpub.com/adt

Submit your paper for peer review online: https://mc.manuscriptcentral.com/adt 